POS $\quad \begin{aligned} & \text { PROCEEDINGS } \\ & \text { OF SCIENCE }\end{aligned}$

\title{
Conference Summary and Outlook: Particle Physics Past, Present, and Future
}

\author{
Paul Langacker \\ Institute for Advanced Study \\ Princeton, NJ USA 08540 \\ E-mail: pgl@ias.edu
}

I briefly summarize highlights of ICHEP2018, comment on the $50^{\text {th }}$ anniversary of the Standard Model, and share some of my thoughts for the future.

The 39th International Conference on High Energy Physics (ICHEP2018)

4-11 July, 2018

Seoul, Korea 


\section{Introduction}

The International Conference on High Energy Physics, or "Rochester Conference," has been held every one or two years since the first one in Rochester, New York in 1950. ICHEP2018, held in Seoul, South Korea, brought together over 1100 participants to discuss all aspects of particle physics, including experiment, phenomenology, formal theory, astro-particle, accelerator, detector, computing, education, diversity, applications, and ties to industry. There were some 835 parallel talks in 16 sections, 41 plenary talks, and 226 posters, as well as satellite meetings, public lectures, award talks, and a Director's panel. I will not attempt to summarize everything at the conference, but will focus on my own view of some of the highlights, as well as a brief overview of the 50 year development of the Standard Model and some thoughts for the future.

\section{Happy $5^{\text {th }}$ birthday Standard Model!}

The ICHEP organizers asked me to begin with a brief sketch of the development of the Standard Model (SM), which celebrated its $50^{\text {th }}$ birthday last Fall.

\subsection{Theoretical preludes (1927-1967)}

The birth of the Standard Model is usually taken to be the publication of Weinberg's $S U(2) \times$ $U(1)$ model of leptons in 1967 [1], but of course that and the subsequent additions incorporated many ideas and experimental facts from earlier. ${ }^{1}$ Some of the most important ingredients were:

- Gauge theories. Key aspects include the quantization of the electromagnetic field in 1927 [5]; the subsequent development of quantum electrodynamics (QED) [Figure 1], which combined quantum mechanics, special relativity, and classical electrodynamics [6]; renormalization theory, which evaded the infinities; Yang-Mills theory [7], which extended gauge invariance from $U(1)$ to a non-abelian group; and axiomatic field theory [8], which clarified such fundamental field-theoretic issues as the CPT theorem.

- The Fermi theory of the weak interactions [9]. Though not a gauge theory, the Fermi interaction (including its subsequent modifications) provided the framework for the $S U(2) \times U(1)$ theory, and is itself an excellent first approximation to a large variety of weak interaction processes.

- The strong interaction approximate symmetries $S U(2)$ (isospin) and $S U(3)$ (the eightfoldway) (e.g., [10, 11]) [Figure 2]; spontaneous symmetry breaking and the Goldstone theorem [12, 13]; quarks [14]; color [15]; and scaling [16, 17].

- The Higgs et al. mechanism [18, 19], which simultaneously evaded the existence of unobserved massless Goldstone bosons in a spontaneously broken gauge theory and allowed nonzero mass for vector bosons.

\footnotetext{
${ }^{1}$ For a more detailed description of the history, see, e.g., [2, 3, 4].
} 


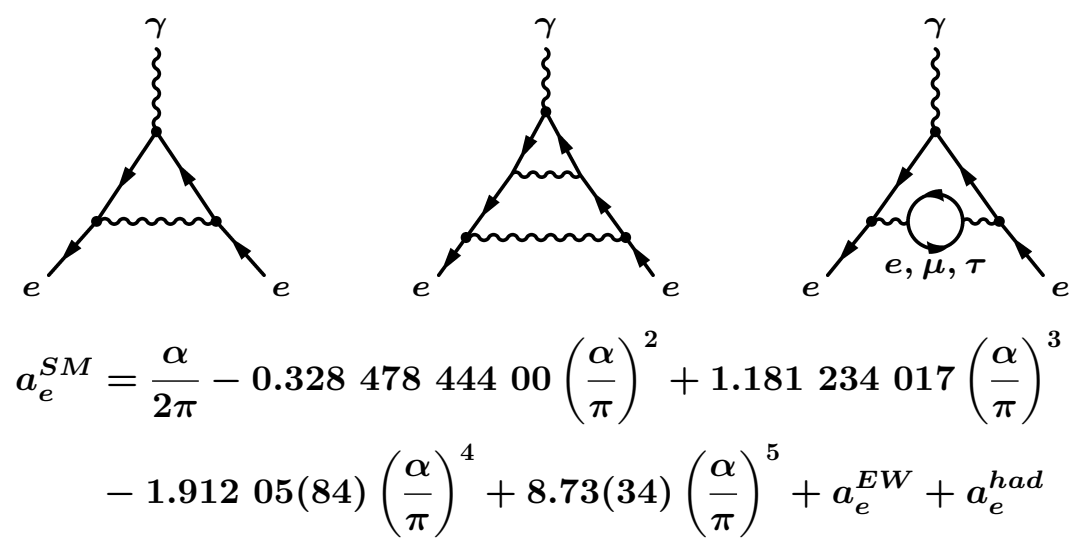

Figure 1: QED has been spectacularly successful. Shown are typical diagrams for the anomalous magnetic moment of the electron, and the SM expression to $\mathscr{O}\left(\alpha^{5}\right)$.
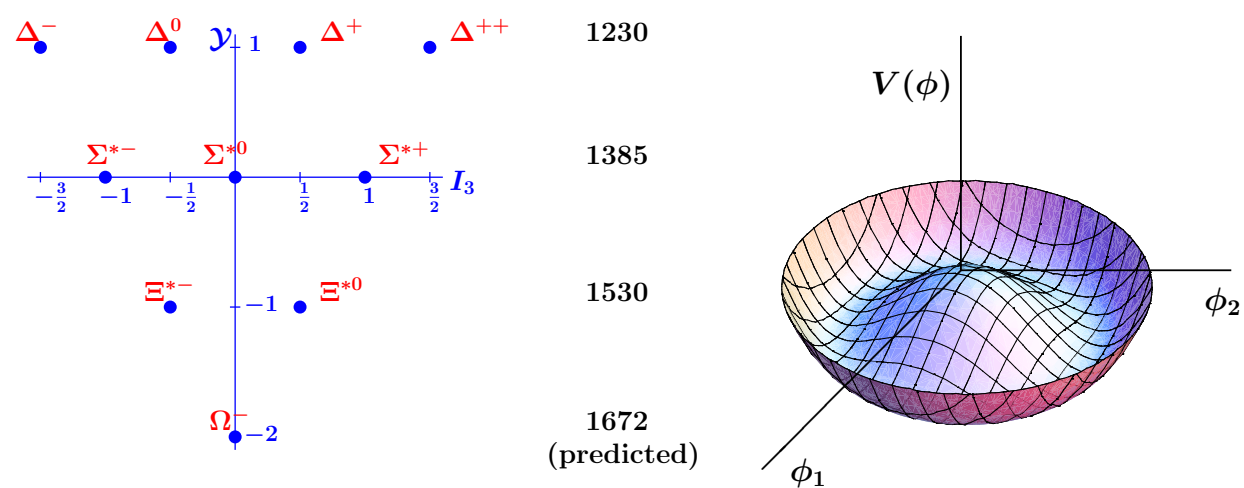

Figure 2: Left: the spin-3/2 decuplet, for which the $\Omega^{-}$existence and mass were successfully predicted by $S U$ (3), and which was also the original motivation for color. Right: the potential for a spontaneously broken $U(1)$ symmetry. The rolling mode around the minimum corresponds to a massless Goldstone boson for a global symmetry. It is "eaten" to become the longitudinal mode of a massive vector boson in a gauge theory. Radial excitations correspond to a massive Higgs-like boson.

\subsection{The $S U(2) \times U(1)$ model of leptons}

The Weinberg-Salam electroweak model $[1,20]$ combined the $S U(2) \times U(1)$ structure, which had been proposed previously [21], with the Higgs mechanism, which gave a satisfactory method for generating gauge boson masses. The Lagrangian density of the model (or any chiral gauge theory) is schematically

$$
\mathscr{L}=-\frac{1}{4} F_{\mu v} F^{\mu v}+\bar{\psi} i \not D \psi+\left(D^{\mu} \phi\right)^{\dagger}\left(D_{\mu} \phi\right)-V(\phi)-\left(\bar{\psi}_{L} \Gamma \psi_{R} \phi+\text { h.c. }\right) .
$$

Notable developments include:

- The original Higgs et al. papers envisioned applications (if any) to the strong interactions, whereas in [1] it was realized that the relevant application was to the weak interactions. 
- Renormalizability, originally conjectured, was established in 1971 [22].

- The original model focussed on leptons because quarks were not at the time well-established. Also, a simple extension involving just three quarks $(u, d, s)$ would have led to unacceptable flavor-changing neutral current (FCNC) effects. However, the gradual establishment of the quark idea and the discovery of charm $(c)$ in 1974 allowed a satisfactory extension, with flavor-changing effects cured by the GIM mechanism [23]. The subsequent discovery of the $\tau$ and $b$, and later the $t$ quark and $v_{\tau}$, implied a third family, allowing a simple origin for $C P$ violation [24].

- The discovery of the predicted weak neutral current (WNC) in 1973, as well as subsequent generations of WNC experiments, and of the $W^{ \pm}$and $Z$ gauge bosons in 1983.

- Precision Z-pole (LEP, SLC) and collider (LEP 2, Tevatron, LHC) experiments (1989-).

- Flavor physics, such as $K$ and $B$ decays and mixing, $C P$ violation, searches for FCNC, and the unitarity triangle.

- The combination of these experimental probes established the basic structure of the $S U(2) \times$ $U(1)$ model, constrained small deviations from many types of new physics, confirmed the program of renormalization, correctly predicted the approximate values of the $t$ and (less accuratedly) Higgs masses, and indicated an approximate unification of the gauge couplings (including the QCD coupling) at a very high energy scale. (The gauge unification is more successful in the supersymmetric extension.) [Figure 3]

- The observation of neutrino mass and mixing effects, ${ }^{2}$ established in atmospheric neutrino oscillations in 1998 [28] (but with strong indications earlier from Solar neutrinos). It is still not certain whether the masses are Dirac or Majorana.

- The observation of the Brout-Englert-Higgs (BEH) or "Higgs" boson by the ATLAS and CMS collaborations in 2012.

\subsection{Quantum Chromodynamics (QCD)}

The strong interaction part of the Standard Model is QCD [Figure 4], which was developed in the early 1970s (e.g., [29]). QCD is a gauge theory based on $S U(3)$. It is essentially the unique renormalizable field theory consistent with the experimental information available at the time. Principal ingredients and developments included:

- Deep inelastic ep scattering [17] and $e^{+} e^{-}$annihilation experiments at SLAC, which established spin-1/2 partons (quarks) and color.

- The notions of asymptotic freedom [30, 31], infrared slavery, and color confinement.

- The observation of the gluon at DESY in 1979.

\footnotetext{
${ }^{2}$ The original Standard Model assumed massless neutrinos, and many authors consider neutrino mass to be "Beyond the Standard Model" (BSM). I choose instead to redefine the Standard Model to include neutrino mass in the same way that it was redefined to included the third family of fermions.
} 

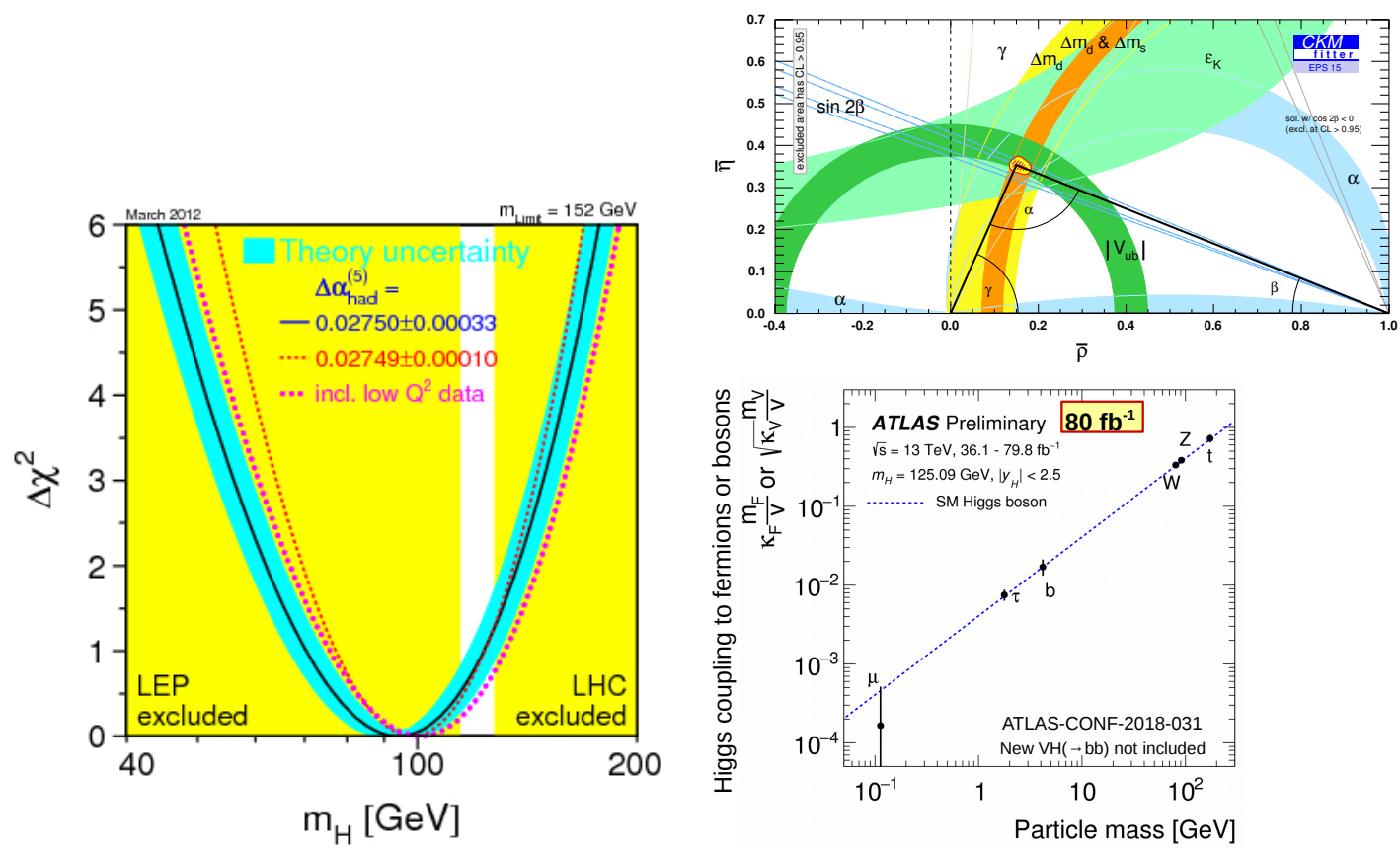

Figure 3: Left: The "blue-band" plot predicting the Higgs mass from indirect precision data, courtesy of the LEP Electroweak Working Group [25] [http:// lepewwg.web.cern.ch/LEPEWWG/]. Top right, the unitarity triangle, courtesy of the CKMfitter group [26] [http://ckmfitter.in2p3.fr/]. Bottom right: observed and predicted Higgs couplings [27].

- Sophisticated theoretical work involving perturbative QCD, jet physics, hadronization, the development of generators, and amplitude methods.

- First principle lattice QCD calculations of the hadron spectrum, confinement, weak matrix elements, etc.

\subsection{Standard Model successes, problems, missing ingredients, and questions}

The Standard Model has been spectacularly successful, far more so than (hardly anyone) anticipated 50 years ago. It is mathematically consistent and describes ordinary matter and interactions to an excellent approximation down to a distance scale of some $10^{-16} \mathrm{~cm}$. It makes many predictions and has successfully passed many experimental tests, often to high precision. Furthermore, its gauge and matter content leads to several accidental symmetries at the perturbative/renormalizable level, such as baryon and lepton number conservation, and the absence of tree-level flavor changing neutral currents and electric dipole moments (EDMs). ${ }^{3}$

Nevertheless, there are a number of problems, missing ingredients, and questions, including:

- There are 27 (29) free parameters for Dirac (Majorana) neutrinos.

- The gauge interactions are very complicated, involving the direct product of three groups with different properties.

\footnotetext{
${ }^{3}$ With the appropriate caveats for some extensions involving Majorana neutrino masses and the strong $C P$ parameter.
} 


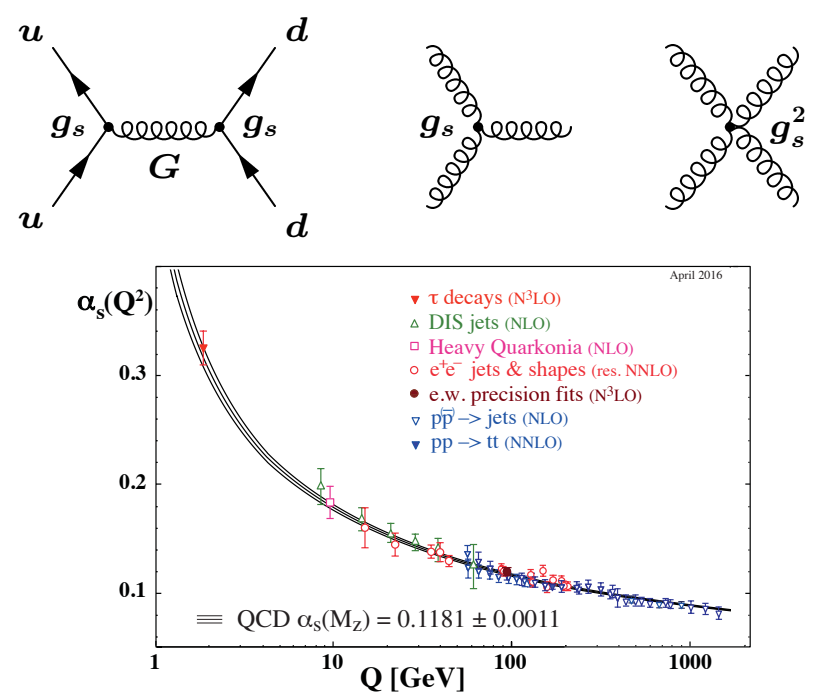

Figure 4: Top: gluon exchange between quarks, and the gluon self-interactions. Bottom: the running QCD coupling, courtesty of the Particle Data Group [http://pdg . lbl . gov].

- There is no complete explanation for charge quantization. ${ }^{4}$

- There is no fundamental explanation for the fermion families, masses, and mixings (including the type and magnitudes of the neutrino masses). These account for most of the free parameters.

- There are severe fine tunings, including the Higgs mass and associated electroweak scale, vacuum energy (cosmological constant), and $\theta_{\mathrm{QCD}}$.

- The Standard Model does not include quantum gravity.

- The initial conditions on the Big Bang.

- The origin of the baryon asymmetry.

- The nature of the dark matter and energy.

- Such conventional paradigms as uniqueness, naturalness, and minimality should possibly be reconsidered.

- Why does Nature appear to be "just right"?

- There are possible experimental deviations from the SM, such as $g_{\mu}-2$, the $B$ decay anomalies, and possible sterile neutrinos.

\footnotetext{
${ }^{4}$ Anomaly cancellation must be supplemented with additional assumptions, such as family universality.
} 


\section{ICHEP2018}

I now turn to a selection of highlights from the conference and of developments in the two years since ICHEP2016.

\subsection{Collider physics}

Although no major discoveries were reported, the technical accomplishments in collider physics were impressive [Figure 5]:

- The LHC and detectors have performed superbly [27, 32]. The luminosity for ATLAS and CMS is around twice the design value, with average pileups around 33! Major detector upgrades have been accomplished, and numberous advances in analysis methods and machine learning have been carried out.

- There have been major programs in perturbative QCD, with most important processes computed to NNLO. There have been corresponding advances in PDFs, jet substructure, generators, amplitude methods, and lattice calculations.

- A wide range of QCD and electroweak processes have been measured, with impressive agreement with Standard Model expectations.

- Phase II of the SuperKEKB/BELLE II project has started running [33], with a luminosity 40 times that of KEK.

\subsection{The Higgs}

The observed $125 \mathrm{GeV}$ boson is consistent with the (minimal) SM Higgs. However, its couplings have only been measured at the 10-25\% level and more complicated scenarios are still possible. These include two or more Higgs doublets (as in supersymmetry), the existence of additional scalar singlets or triplets (as in many models of electroweak baryogenesis or low-scale neutrino seesaws), or composite Higgs models.

ATLAS and CMS have continued to refine their measurments of the BEH boson mass, couplings, and properties [34] [Figures 3 and 6]. In particular, the $t \bar{t} H, b \bar{b} H$, and $\tau^{+} \tau^{-} H$ Yukawa couplings have been observed at more than $5 \sigma$, consistent with the SM, and a non-trivial upper limit placed on $H \rightarrow \mu^{+} \mu^{-}\left(\sigma<2.1 \sigma_{\mathrm{S} M}\right)$. All major production modes have been observed, ATLAS has set a new indirect limit of $14.4 \mathrm{MeV}$ on the width (vs. $4.1 \mathrm{MeV}$ in the SM), $p_{T}$ and other distributions have been measured, and limits have been set on additional Higgs particles.

Alternatives/extensions to the minimal model often predict deviations in the couplings at the $1-10 \%$ level. The HL-LHC and proposed future $e^{+} e^{-}$and $p p$ colliders are expected to have $\mathscr{O}(1 \%)$ precision, and may be able to measure the induced $H^{3}$ vertex (which is especially sensitive to new physics) and the total width (via $e^{+} e^{-} \rightarrow Z^{*} \rightarrow Z\left[H \rightarrow Z Z^{*}\right]$, combined with branching ratios). 

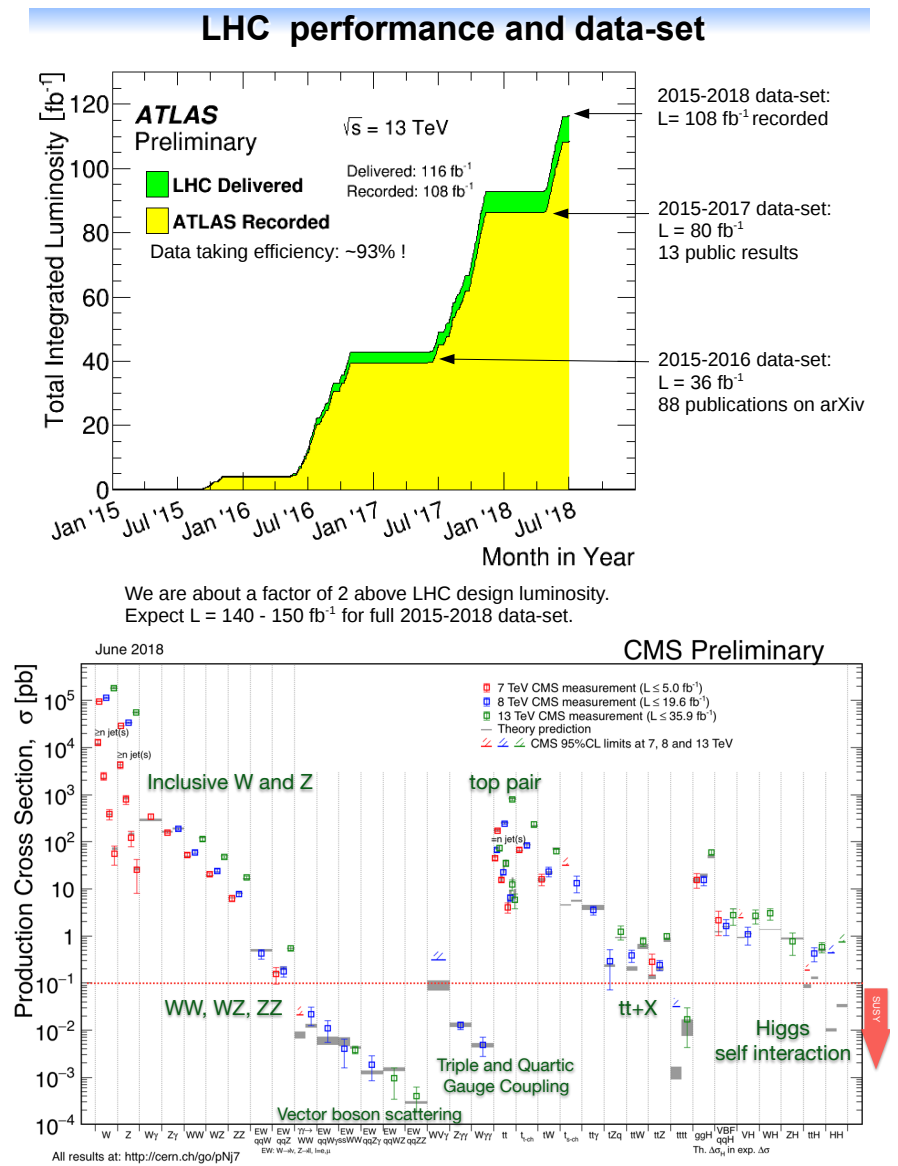

Figure 5: Top: LHC luminosity [27]. Bottom: Observed cross sections compared with SM expectations [32].

\begin{tabular}{|c|c|}
\hline \multicolumn{2}{|c|}{ Yukawa coupling to fermions: } \\
\hline Top-quark: ttH & $80 \mathrm{fb}^{-1}$ \\
\hline $6.3 \sigma(5.1 \sigma)$ obs (exp) & arXiv:1806.00425 \\
\hline Beauty-quark $\mathrm{H} \rightarrow$ bb: & $80 \mathrm{fb}^{-1}$ ₹New\} \\
\hline $5.4 \sigma(5.5 \sigma)$ obs $(\exp )$ & ATLAS-CONF-2018-036 \\
\hline Tau-lepton: $\mathrm{H} \rightarrow \tau \tau$ & \\
\hline $6.4 \sigma(5.4 \sigma)$ obs (exp) & ATLAS-CONF-2018-021 \\
\hline Muon $\mathrm{H} \rightarrow \mu \mu$ : & $80 \mathrm{fb}^{-1}$ \{News \\
\hline$\sigma_{\text {limit }} / \sigma_{\mathrm{sM}}<2.1$ (obs) & ATLAS-CONF-2018-026 \\
\hline $\begin{array}{l}\text { Charm-quark: } \mathrm{H} \rightarrow \mathrm{cc} \text { : } \\
\sigma_{\text {limit }} / \sigma_{\mathrm{SM}}<104 \text { (obs) }\end{array}$ & PRL 120 (2018) 211802 \\
\hline
\end{tabular}

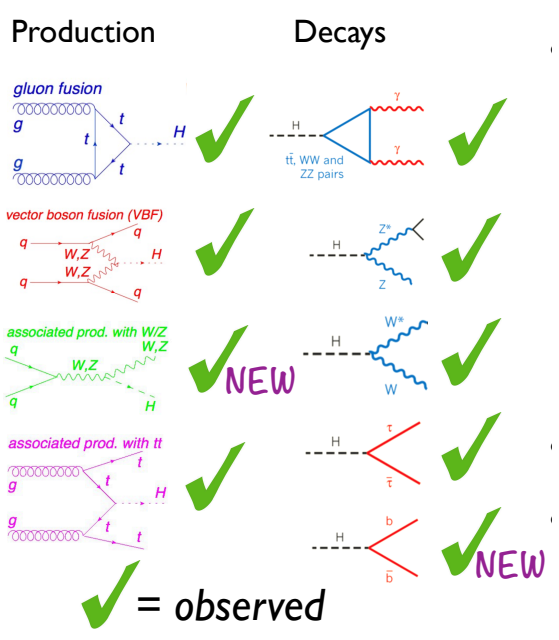

Figure 6: Left: Higgs Yukawa couplings (ATLAS) [27]. Right: Higgs production and decay modes [34]. 


\subsection{Heavy ions}

Heavy ion collsions $[35,36,27]$ explore the physics of QCD at extreme temperatures and densities, with implications for QCD itself as well as for conditions in the early Universe (the "quark-gluon plasma" or QGP) and in dense astrophysical objects.

There has been an extensive experimental program at RHIC at Brookhaven, and by the ALICE, CMS, and ATLAS experiments at the LHC [Figure 7]. The LHC running has extensively studied hot ("fireball") QGP effects in $P b-P b$ collisions (and some recent running of $X e-X e$ ), as well as using $p p$ and $p-P b$ data as baselines to separate ordinary hadronic and nuclear effects. Observations include the (very large) multiplicities as functions of the centrality of the collision, $p_{T}$, and particle type; jet quenching and propagation of heavy flavors; long-range correlations; and azimuthal flows. The results imply that under existing collider conditions the final state is neither a collection of hadrons nor an ideal gas of quarks and gluons. Rather, there are strong collective effects more characteristic of a smoothly flowing fluid.
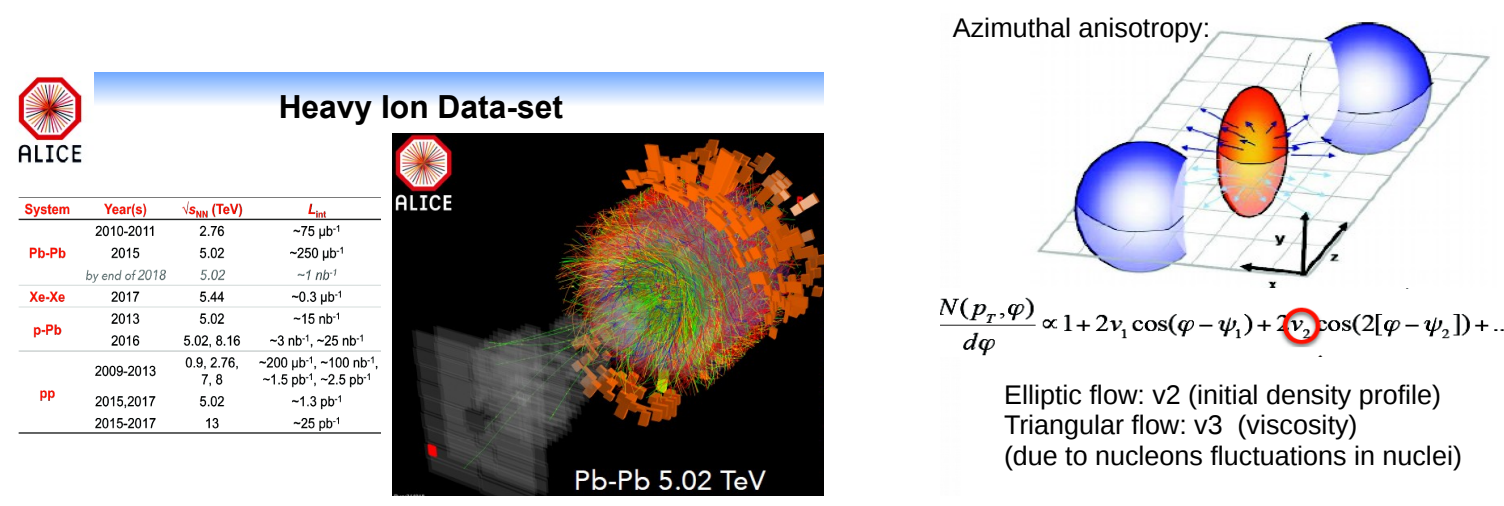

Figure 7: Left: ALICE heavy ion data [27]. Right: Azimuthal flows [27].

\subsection{Electroweak and top}

Electroweak measurements at the LHC [37] include precise determinations of the leptonic weak angle $\sin ^{2} \theta_{e f f}^{\ell}$ from asymmetries in dilepton production (especially important because of the discrepancy between the most precise $Z$-pole determinations) [Figure 8]; $W, Z$, and $\gamma$ production (as tests of QCD, $M_{W}$, etc.); $W W, W Z$, and other diboson productions (as tests of triple gauge vertices); and diboson scattering (as tests of unitarity, the electroweak symmetry breaking mechanism, and quartic gauge vertices).

Top quarks are produced prolifically in $t \bar{t}$ pairs (180 million so far at the LHC [38]), as well as singly or in association with other particles [37, 38]. Measurements of cross sections, spin correlations, etc. are important as QCD tests, for a precise $m_{t}$ (needed for precision electroweak and Higgs physics), determination of $V_{t b}$, and searches for new physics (such as in rare flavorchanging decays). High precision calculations of SM expectations, such as NNLO QCD and NLO EW, are essential to match the experimental precision [38]. 


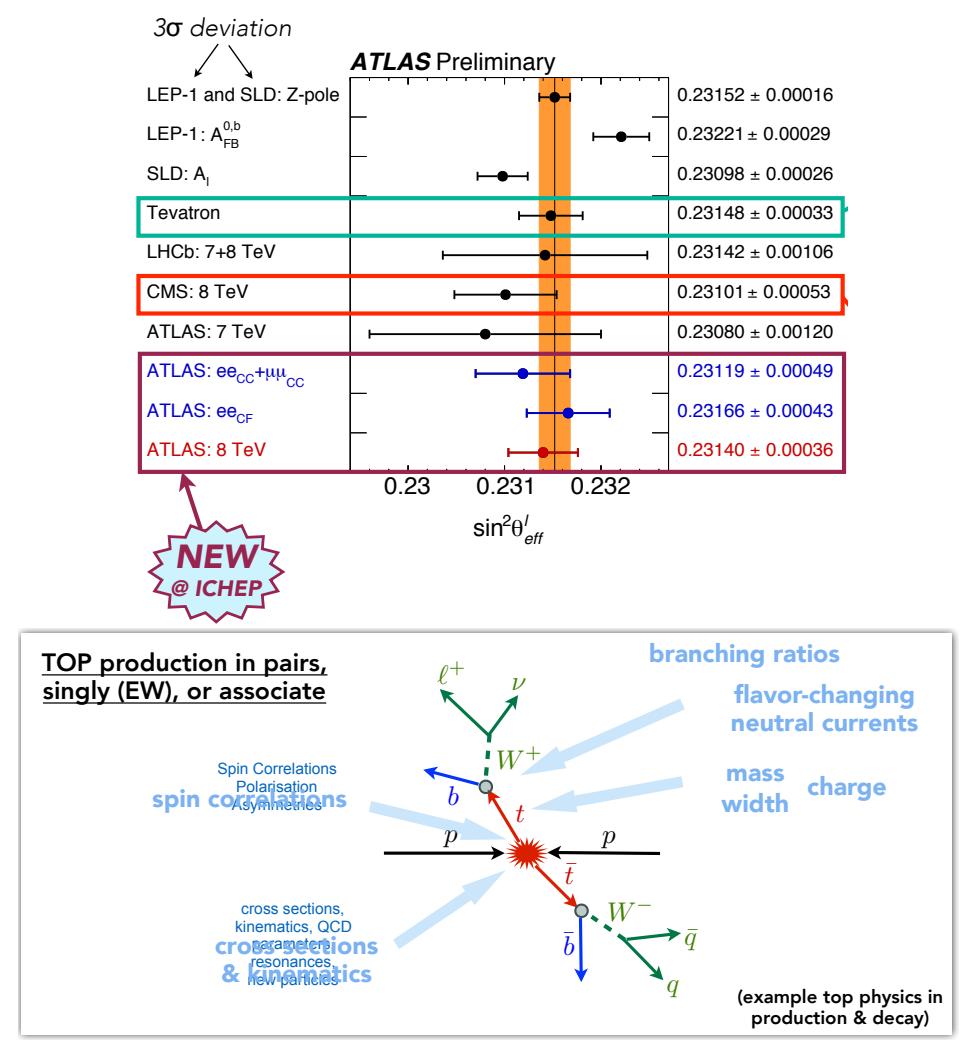

Figure 8: Top: Measurements of the weak angle [37]. Bottom: Top-pair production [37].

\subsection{Exotic searches}

Searches for exotics [39] include vector (non-chiral under the SM) fermion pairs, such as heavy charge-2/3 quarks $T-\bar{T}$ decaying into ordinary quarks plus $W, Z$, or $H$; heavy resonances, such as $Z^{\prime}, W^{\prime}$, colorons, etc. (searched for in dilepton, dijet, diboson, or $t \bar{t}$ spectra); weakly coupled or long-lived particles; dark matter; leptoquarks (suggested in some explanations of the $B$ anomalies); diquarks; and particles that could be portals to a dark sector, such as extra Higgs particles or light weakly-coupled vectors. Some of these, such as vector fermions or $Z^{\prime}$ emerge in many types of new physics (e.g., as string remnants or in composite Higgs models), which is both an advantage (more likely to occur) and disadvantage (harder to diagnose if observed) [Figure 9].

New or improved search techniques include boosted jets/jet substructure, associated production, and mono-particles associated with missing transverse energy.

\subsection{Supersymmetry}

Supersymmetry has long been one of the most popular extensions of the SM, motivated by the Higgs naturalness problem, gauge coupling unification, and its prediction of a plausible WIMP candidate for cold dark matter. Furthermore, the Coleman-Mandula theory asserts that under plausible assumptions supersymmetry is the unique space-time extension of the Poincaré algebra. Finally, supersymmetry is required in realistic string constructions (although the supersymmetry breaking scale could be large). 


\begin{tabular}{|c|c|}
\hline examples & Mass Lower limit \\
\hline String resonance (jj) & $\sim 8 \mathrm{TeV}$ \\
\hline Excited quark (jj) & $\sim 6 \mathrm{TeV}$ \\
\hline Z' (SSM) (II) $^{\prime}$ & $\sim 4.5 \mathrm{TeV}$ \\
\hline W' (SSM) (Iv) & $\sim 5.5 \mathrm{TeV}$ \\
\hline
\end{tabular}

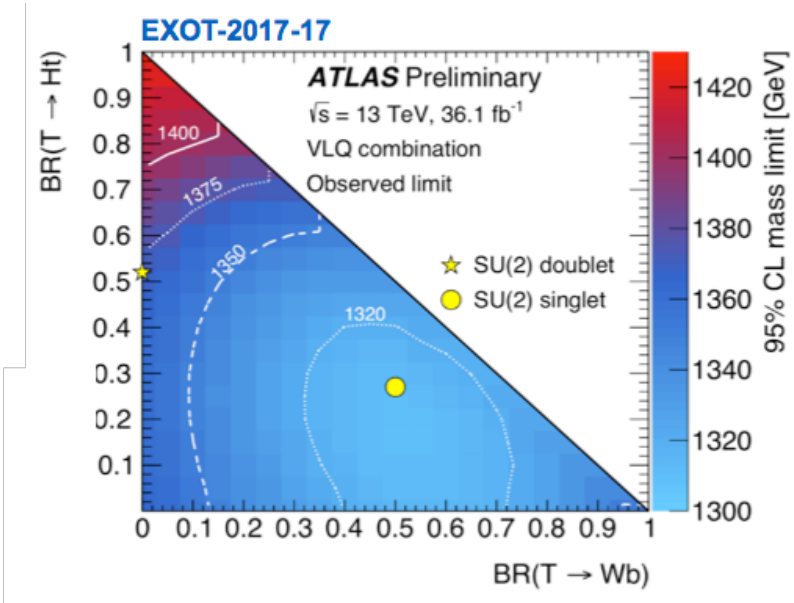

Figure 9: Left: Limits on resonances [39]. Right: $T \bar{T}$ pairs [39].

There are many versions of supersymmetry, even within the minimal model (MSSM). Early experimental searches focussed on favorable scenarios, e.g., involving large missing $E_{T}, R$-parity $\left(R_{P}\right)$ conservation, etc., and often utilized specific supersymmetry-breaking models, or simplified models in which there was a single decay mode. Since no signals have been observed, most recent analyses ${ }^{5}$ [40] have concentrated on more challenging possibilities, e.g., with less missing $E_{T}$, heavy neutralinos, multiple decays modes, or longer decay chains. These are often analyzed in the framework of the much more general phenomenological MSSM (pMSSM), or in specific scenarios such as compressed, stealth, split, light gravitino (gauge mediation), $R_{P}$ violation, etc. There have also been many more studies of electroweak sparticles and of the third sfamily, which have lower production cross sections.

So far, there is no sign of supersymmetry [Figure 10]. A rough summary of the current exclusions under favourable/challenging scenarios is [40]:

- gluinos up to $\mathscr{O}(2) / \mathscr{O}(1) \mathrm{TeV}$.

- Squarks up to $\mathscr{O}(1.5) / \mathscr{O}(0.5) \mathrm{TeV}$.

- Stops and sbottoms up to $\mathscr{O}(1) / \mathscr{O}(0.7) \mathrm{TeV}$.

- EW produced sparticles up to $\mathscr{O}(0.5-1) / \mathscr{O}(0.1) \mathrm{TeV}$.

Although supersymmetry may still very well turn up in future analyses and runs, the lack of evidence for this or many other popular extensions of the SM is beginning to challenge the naturalness paradigm. Also, a higher scale for the superpartner masses makes it difficult to explain the $g_{\mu}-2$ anomaly using supersymmetry. On the other hand, it somewhat relaxes the tension from the nonobservation of FCNC or EDMs and makes it easier to accomodate the observed Higgs mass (which is a bit large for much of the MSSM parameter space).

\footnotetext{
${ }^{5}$ Most of the results presented at ICHEP2018 utilize $\sim 36 \mathrm{fb}^{-1} /$ detector of $13 \mathrm{TeV}$ data from 2015-2016, with some using $80 \mathrm{fb}^{-1}$ from 2015-2017.
} 
There are also many plausible extensions of the MSSM, e.g., involving additional $S U(2)$ singlet Higgs fields (the NMSSM and generalizations) or the UMSSM (involving an additional $Z^{\prime}$ ), which often relax the naturalness issue.

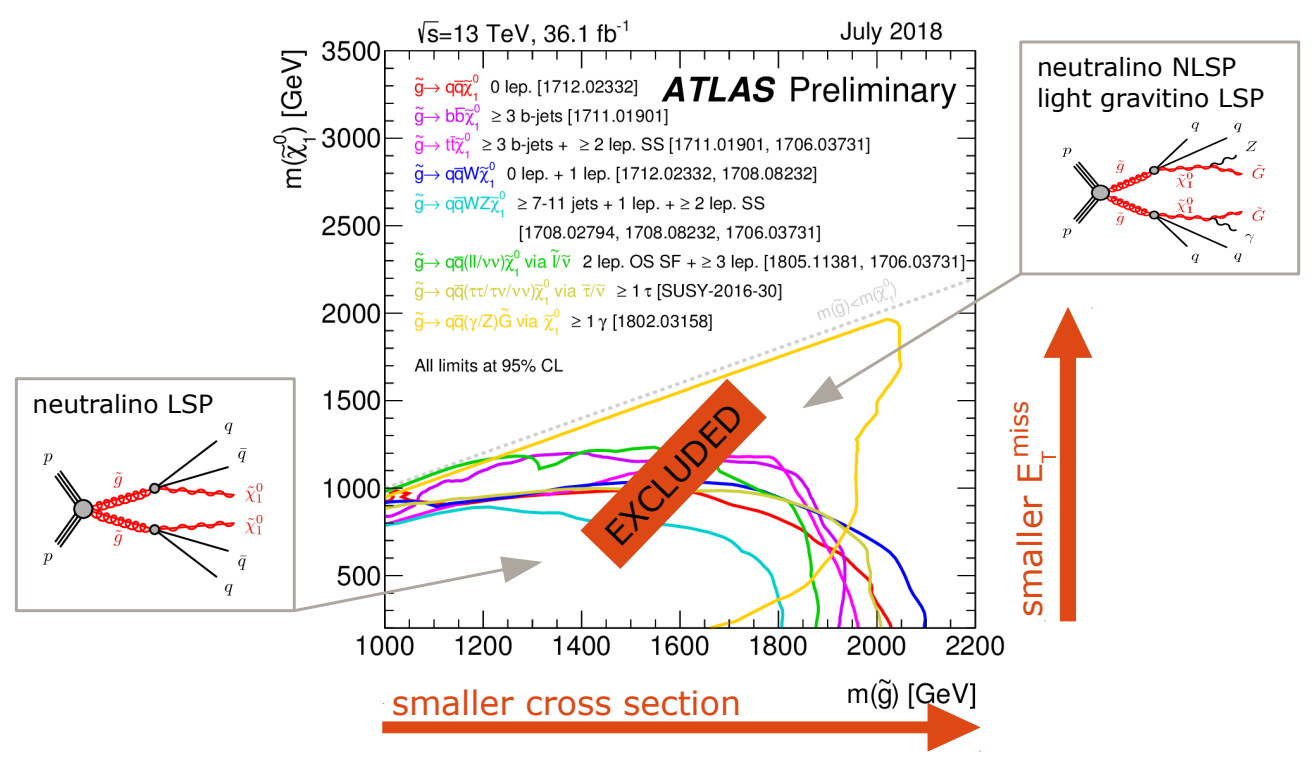

Figure 10: Typical exclusions in the MSSM [40].

\subsection{Flavor physics}

\section{$C P$ violation and the CKM matrix}

One of the most interesting new results is the more precise determination of the unitarity triangle angle $\gamma\left(\phi_{3}\right)$, dominated by a number of new and updated results from LHCb [32, 41] [Figure 11]. Another is that the long-standing discrepancy between the exclusive and inclusive determinations of $\left|V_{c b}\right|$ has been resolved by a new Belle determination from $B \rightarrow D^{*} \ell v$, analyzed in a framework with minimal theoretical assumptions and utilizing measured form factors [42] [Figure 11]. Hopefully, similar progress will be made on the similar $\left|V_{u b}\right|$ discrepancy in the future.

\section{Flavor anomalies}

The flavor anomalies [32, 43, 44] in the ratios

$$
R_{D^{(*)}}=\frac{B F\left(B \rightarrow D^{(*)} \tau v\right)}{B F\left(B \rightarrow D^{(*)} \mu v\right)} \quad \text { and } \quad R_{K^{(*)}}=\frac{B F\left(B \rightarrow K^{(*)} \mu^{+} \mu^{-}\right)}{B F\left(B \rightarrow K^{(*)} e^{+} e^{-}\right)}
$$

if confirmed, would imply a breakdown of lepton family universality. There is also a suggestion of new physics in the $B \rightarrow K^{*} \mu^{+} \mu^{-}$angular distributions, best described by an effective $V-A$ coupling to the quarks and $V$ or $V-A$ to the leptons.

$R_{D^{(*)}}$ suggests a relatively large new physics effect since the SM decays occur at tree level, while new physics explanations for $R_{K^{(*)}}$ only have to compete with (small) SM penguins. Many authors have suggested the possibility of a leptoquark to account for $R_{D^{(*)}}$, while either a leptoquark 

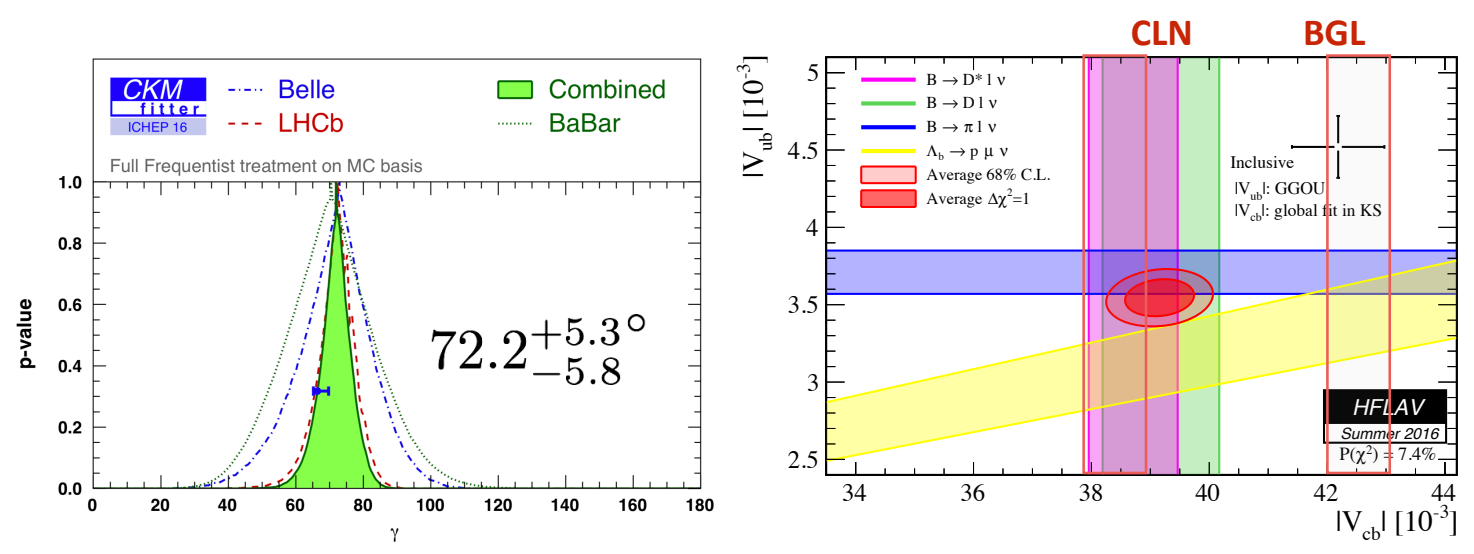

Figure 11: Left: The $C P$ violation angle $\gamma\left(\phi_{3}\right)$ [32]. Right: determinations of $\left|V_{c b}\right|$ and $\left|V_{u b}\right|$ [42].

or flavor-violating $Z^{\prime}$ have been suggested for $R_{K^{(*)}}$. Constaints on some specific models are shown in Figure 12.
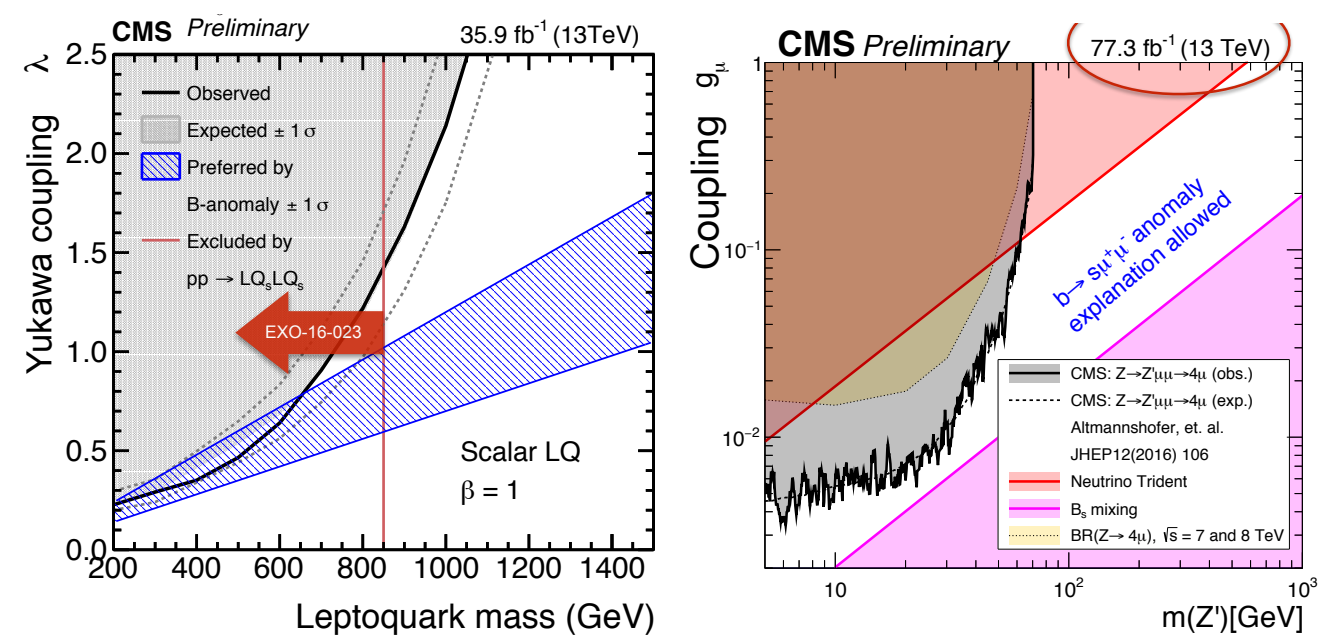

Figure 12: Constraints on leptoquark (left) and $Z^{\prime}$ (right) models suggested by the $B$ anomalies [32].

\section{Other important results, limits, prospects, or anomalies}

Many other important topics in flavor physics were discussed [27, 32, 43, 44, 45], including:

- $B$ and $D$ decays; $C P$ violation; and the CKM matrix.

- $B_{s} \rightarrow \mu^{+} \mu^{-}, B \rightarrow \mu^{+} \mu^{-}$.

- $K^{+} \rightarrow \pi^{+} v \bar{v}, K^{0} \rightarrow \pi^{0} v \bar{v}$.

- $\varepsilon^{\prime} / \varepsilon$.

- $g_{\mu}-2$ 
- Lepton-flavor violation in $\mu \rightarrow e \gamma, \mu \rightarrow 3 e, \mu \rightarrow e$ conversion.

- The muonic Lamb shift (aka the proton radius).

- Electric dipole moments.

- The neutron lifetime discrepancies.

\subsection{Strong interactions}

There has been tremendous progress in lattice QCD, with applications to weak interaction decay constants, form factors, and mixing; hadronic vacuum polarization and light-by-light scattering relevant to $g_{\mu}-2$; neutrino, muon, and dark matter interactions with nucleons and nuclei; and parton distribution functions [46].

There has been extensive experimental work on exotic hadrons, such as pentaquarks and tetraquarks. There are still many theoretical models, but anticipated experimental results should help clarify our understanding [47] [Figure 13].

Experimental programs at Jefferson $\mathrm{Lab}\left(e^{-} N, \gamma N\right)$ and elsewhere are probing parton distributions, the spin structure, and other aspects of the nucleon. There has been a major effort to map the three-dimensional structure of the nucleon, as described by generalized parton distributions (GPDs) [48] [Figure 13].

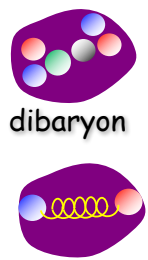

hybrid
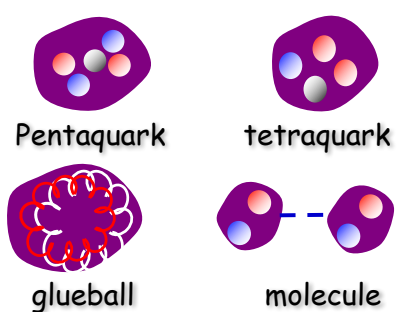

molecule

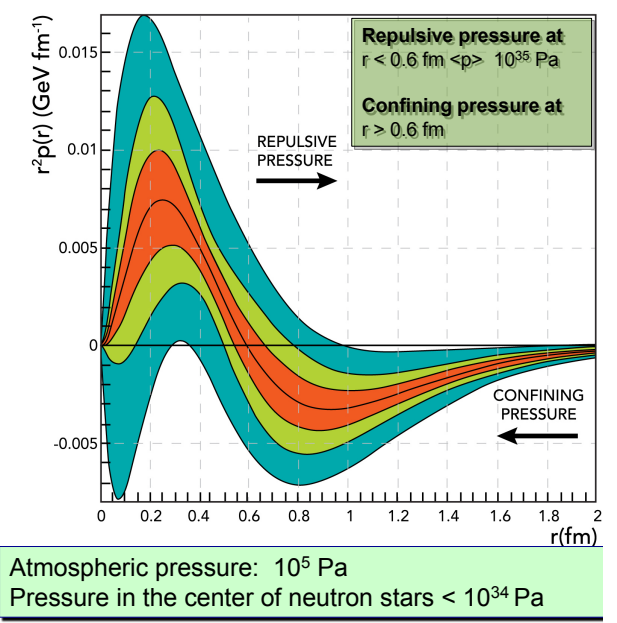

Figure 13: Left: Exotic hadrons [47]. Right: Pressure distribution in the proton, obtained from GPDs [48].

\subsection{Neutrino physics}

Neutrinos are an important probe of (and are constrained by) particle physics, astrophysics, and cosmology.

\section{The standard 3-neutrino picture}

- Recent results within the 3-neutrino picture [49, 50, 52] include a measurement of $\theta_{13}$ from Double Chooz, consistent with (but less precise than) previous Daya Bay and RENO results; 
final $v_{\tau}$ results from OPERA, with 10 candidate events; final results from MINOS/MINOS+; new T2K and NOvA results (including the first data from the NOvA $\bar{v}$ beam). The long baseline data indicate that the normal hierarchy is favored, a nonzero Dirac $C P$ violation phase is favored, and that the upper octant for $\theta_{23}$ is favored over maximal mixing or the lower octant [49] [Figure 14].
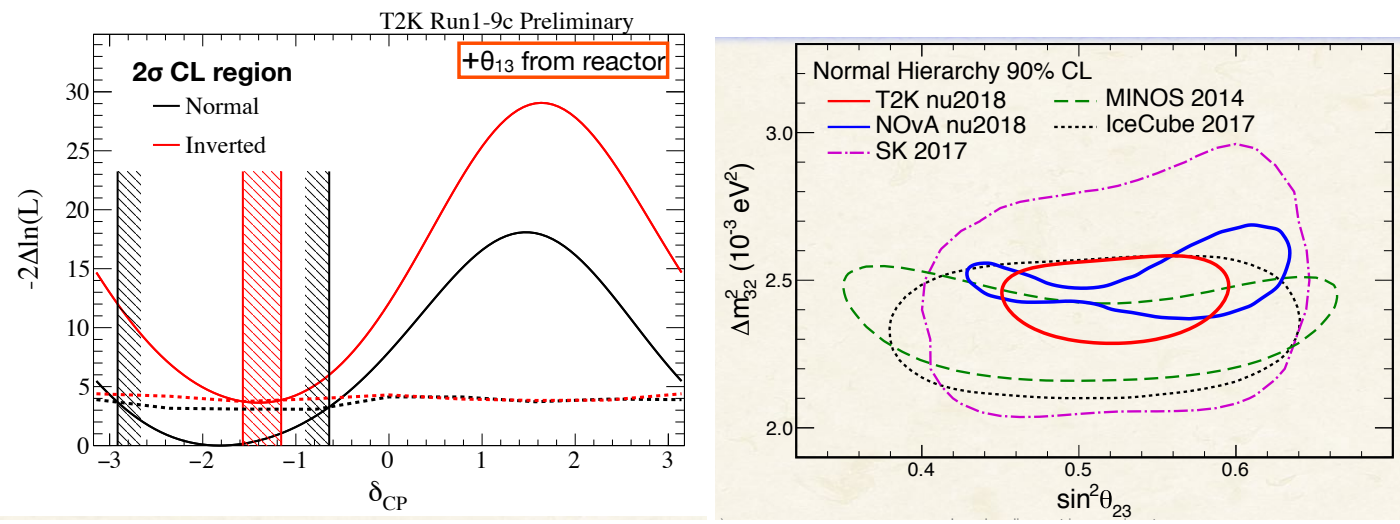

Figure 14: Left: The Dirac neutrino $C P$ phase [49]. Right: $\Delta m_{32}^{2}$ vs. $\sin ^{2} \theta_{23}$ [49].

- In addition to confirming the oscillation results, it is still to be established whether the neutrinos are Majorana (e.g., as in some version of the seesaw model), with implications for leptogenesis, or Dirac (e.g., because the Yukawa couplings are forbidden to leading order by a new symmetry). Similarly, the observed mixings could be associated with a new broken symmetry [50], or could be essentially anarchic (suggestive of superstring theories).

- Accurate measurements of low energy neutrino cross sections are essential for the interpretation of oscillation and other experiments. The Fermilab program includes new MINER $v$ A $\bar{v}$ data on a number of nuclei, and the Short Baseline Neutrino program (SBN), consisting of three liquid argon detectors: MicroBooNE (running), ICARUS, and SBND [51].

- The COHERENT experiment has utilized the Oak Ridge Spallation Neutron Source to make the first measurement of coherent elastic neutrino scattering from the entire nucleus [52] [Figure 15].

- Neutrinosless double beta decay $\left(\beta \beta_{0 v}\right)$ is the only known practical way to probe the Majorana vs. Dirac nature of the light neutrinos. A number of experiments are now sensitive down to around $0.1 \mathrm{eV}$ on the effective $\beta \beta_{0 v}$ mass (with nonnegligible uncertainly from nuclear matrix elements). This is somewhat above the expected range for the inverted mass hierarchy, but excludes much of the degenerate region for Majorana neutrinos [Figure 15]. The experiments should eventually be senstive to around $0.01 \mathrm{eV}$, covering the entire inverted hierarchy region. Unfortunately, probing the region expected for Majorana neutrinos with a normal hierarchy is probably impossible without novel or radical new ideas and a consolidated worldwide effort [52]. 
- The KATRIN trititum $\beta$ decay experiment in Karlsruhe has started running, with a projected sensitivity of $0.2 \mathrm{eV}$ on the effective $\beta$ decay mass [52].

- Cosmological constraints on the sum of the active neutrino masses should eventually be senstive down to around $0.05 \mathrm{eV}$ (the smallest value allowed by the oscillation experiments) or even better [53].

- Many other results were presented on Solar, supernova, atmospheric, high energy, relic, and geo neutrinos.
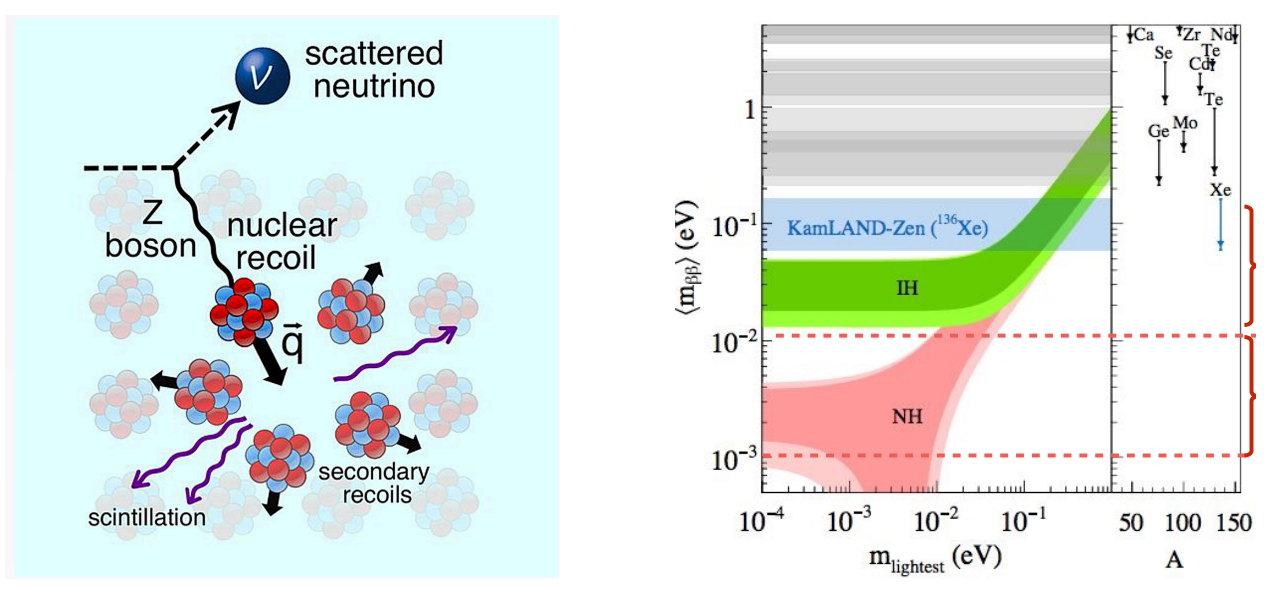

Figure 15: Left: Coherent elastic $v$-nucleus scattering [52]. Right: "Lobster-claw" diagram of the effective $\beta \beta_{0 v}$ mass vs. the lightest mass [52].

\section{Possible sterile neutrinos}

Most models of neutrino mass involve sterile (aka right-handed or singlet) neutrinos, which may be light (as in Dirac neutrinos) or very heavy (as in seesaw models). However, the sterile neutrino-induced oscillations suggested by LSND would require mixing between ordinary and sterile neutrinos of the same helicity, which is nontrivial to achieve. (It would require two types of small mass terms, usually Dirac and Majorana.)

MiniBooNE has doubled its neutrino data set. An oscillation fit to the neutrino and antineutrino data for $\stackrel{(-)}{v}_{\mu} \rightarrow \stackrel{(-)}{v}_{e}$ yields results consistent with LSND, suggesting an $\sim 1 \mathrm{eV}$ sterile neutrino ${ }^{6}$ [54] [Figure 16]. On the other hand, the evidence for sterile neutrinos from the reactor anomaly is weakened by the Daya Bay and RENO measurements of the time dependence of the reactor fluxes, suggesting that the deficit of $\bar{v}_{e}$ is associated with those from ${ }^{235} U$ but not ${ }^{239} \mathrm{Pu}$ [52]. There is also a strong conflict with disappearance experiments [Figure 16] and with cosmological constraints (unless one invokes nonstandard cosmologies). There is currently no coherent picture of what is going on. Hopefully, the situation will soon be clarified by a new generation of short baseline accelerator (SBN), reactor (DANSS, NEOS, PROSPECT, STEREO, SoLid), and source experiments [54].

\footnotetext{
${ }^{6}$ However, the low-energy $v_{e}$ data is not well described by the oscillation hypothesis [Figure 16], emphasizing the importance of the cross section measurements.
} 

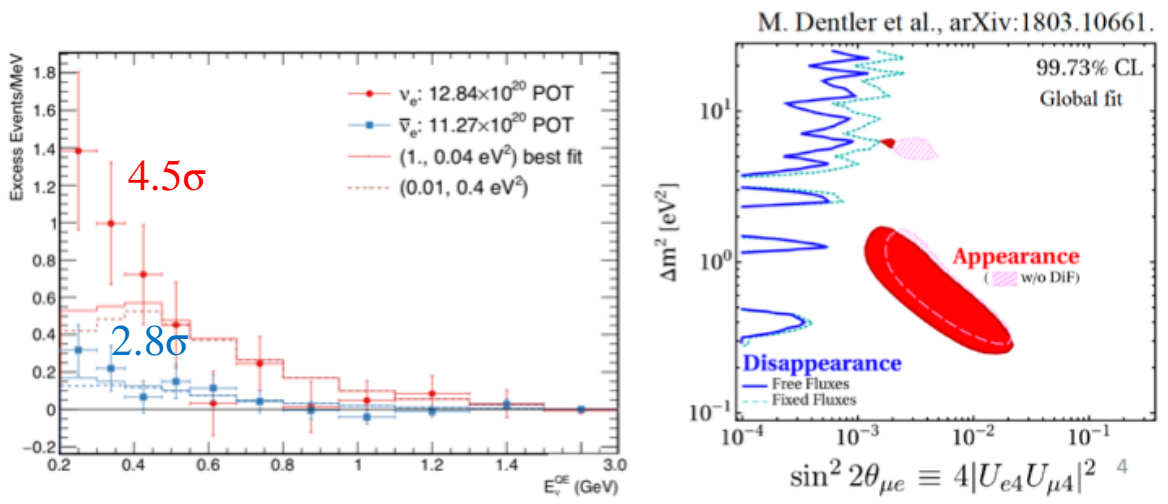

Figure 16: Left: MiniBooNE spectrum [54]. Right: Sterile neutrino appearance (signal) and disappearance (exclusion) regions [54].

\subsection{Dark matter}

There are numerous candidates for dark matter [55]. These include primordial black holes (which are strongly constrained by astrophysics, but may be possible in the mass range observed by LIGO) and many possibilities for particle dark matter. The latter include Weakly Interacting Massive Particles (WIMPs), dark sector particles, light gravitinos (as in gauge mediation), WIMPZILLAs (extremely heavy particles, e.g., $10^{10} \mathrm{GeV}$ ), fuzzy dark matter [extremely light bosons with de Broglie wavelength $\mathscr{O}(\mathrm{kpc})$ ], warm dark matter (e.g., keV-scale sterile neutrinos), moduli, and axions or axion-like particles (ALPs). Dark matter could also be self-interacting or involve two or more components. Another (non-dark matter) possibility is Modification of Newtonian Gravity (MOND), which, however, has difficulty with large scale effects and the Bullet cluster.

There have been numerous direct [56] (interacting with target) and indirect [57] (observation of annihilation or decay products) searches for dark matter [Figure 17], with no confirmed signal. ${ }^{7}$ The direct detection WIMP experiments are approaching the neutrino background floor (from Solar and atmospheric neutrinos), which will limit further progress without dedicated techniques such as directionality. Very light WIMPs are also possible. Possible loopholes in the interpretation include a local variation in the dark matter density and multi-component dark matter.

There have also been extensive searches at the LHC for the production of (unobserved except as missing transverse energy) dark matter particles in association with a jet, $\gamma, W, Z$, Higgs, ... [39].

Axions are moltivated not only by the strong $C P$ problem but also by string theory. The ADMX cavity experiment is starting to probe the strong $C P$ parameter region, and future experiments are expected to reach most of the interesting region [58] [Figure 17].

\subsection{Cosmic rays}

There has been extensive observational work on high energy cosmic rays, up to some $10^{12}$ $\mathrm{GeV} /$ particle [57]. Much of this is focussed on astrophysical issues, such as their origin and composition. More relevant for particle physics are the fluxes of $e^{+}, \bar{p}$ and other antiparticles, which

\footnotetext{
${ }^{7}$ The DAMA/LIBRA signal from an annual modulation is statistically overwhelming $(12.9 \sigma)$, but other experiments exclude a standard WIMP interpretation. Clarification is greatly desired.
} 

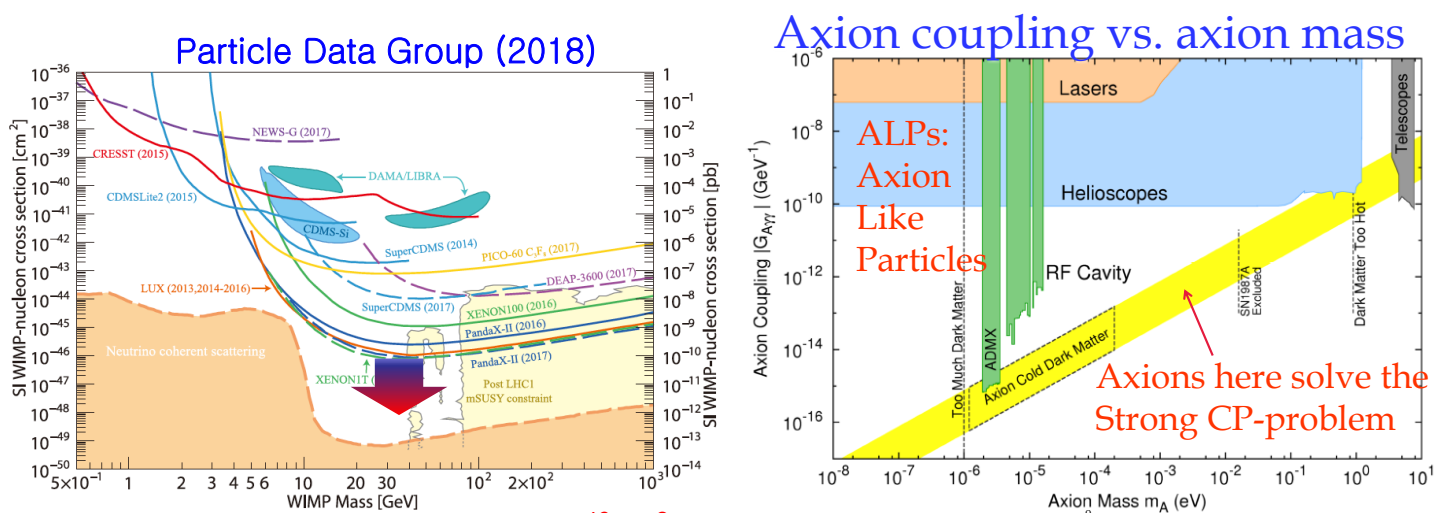

Figure 17: Left: Limits on spin-independent WIMPs. Also shown are the neutrino floor and the allowed region from a minimal supersymmetric model [56]. Right: Constraints on axions [58].

could be produced by dark matter anihilation, by the collisions of ordinary cosmic rays with interstellar matter, or from such astrophysical sources as pulsars. Recent precise data on high energy $e^{+}$and $\bar{p}$ from the Alpha Magnetic Spectrometer (AMS) indicates considerably more antiparticles than predicted by previous estimates of the secondary production rate, but is consistent with the annihilation of TeV-scale dark matter [57] [Figure 18].
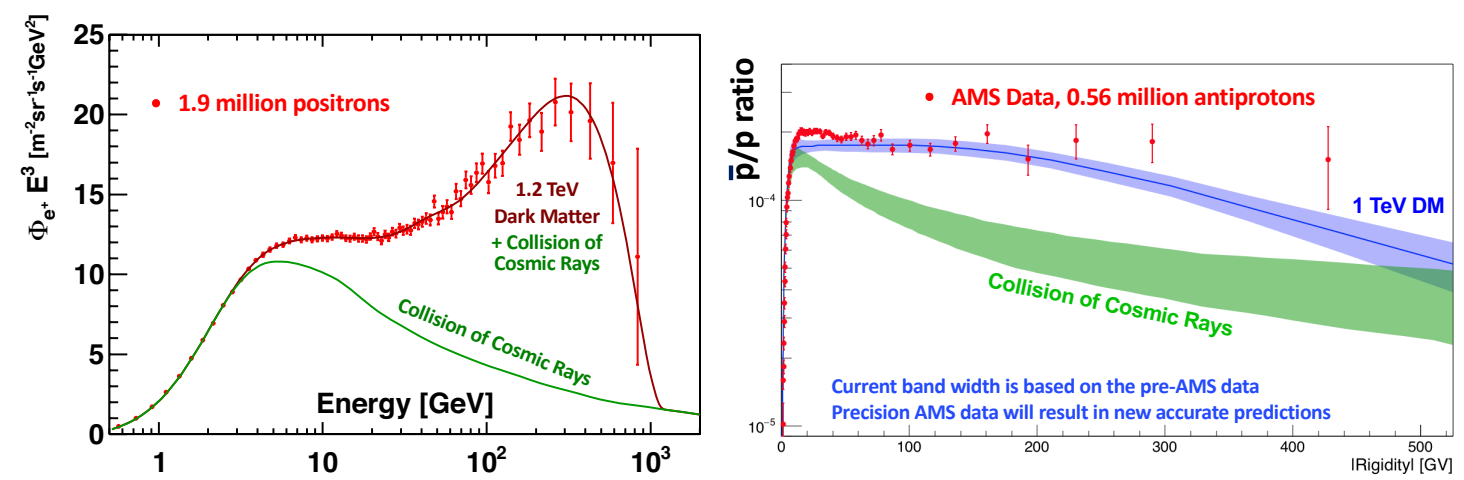

Figure 18: Recent AMS observations of $e^{+}$and $\bar{p}$ [57].

High energy neutrinos have been extensively studied by the IceCube collaboration. ${ }^{8}$ They have recently observed two double cascade events [60], consistent with $v_{\tau}$. This would imply a neutrino flavor ratio $v_{e}: v_{\mu}: v_{\tau} \sim 1.05: 1.35: 0.6$, consistent with $1: 1: 1$ expected from a pion decay source after taking oscillations into account.

\subsection{Gravity waves}

The LIGO and Virgo interferometers have initiated a whole new field in astrophysics, general relativity, and particle physics. The observed binary black hole (BH-BH) mergers involved black

\footnotetext{
${ }^{8}$ Note added: after the conference IceCube announced the observation of a $0.29 \mathrm{PeV}$ event coincident in direction with a known $\gamma$-ray blazar, initiating an extensive multi-wavelength observation program [59].
} 
holes in the $\mathscr{O}\left(10 M_{\odot}\right)$ range and tested GR, e.g., setting an upper limit of $1.2 \times 10^{-23} \mathrm{eV}$ on the graviton mass [61]. The neutron star (NS-NS) merger GW170817 led to a major multi-messenger observation program including a $\gamma$ ray burst and multiple electromagnetic wavelengths [62] [Figure 19]. Implications include the possibility of using such mergers as a standard siren; that they are a site for the $r$-process for the synthesis of elements heavier than ${ }^{56} \mathrm{Fe}$; that the velocity of a gravitational wave relative to light is constrained by $-3 \times 10^{-15}<\beta_{G}-1<7 \times 10^{-16}$; and that some dark energy models are excluded.

Future gravitational wave results are expected to have particle physics implications for cosmological phase transitions, topological defects, and inflation.

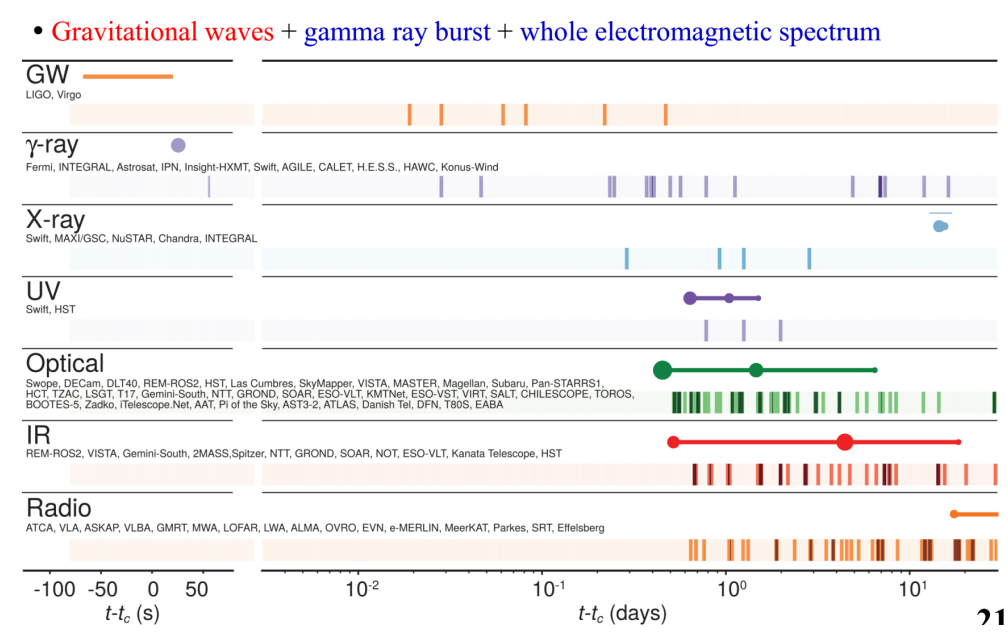

Figure 19: Multi-messenger observations from GW170817 [62].

\subsection{Other topics}

Many other important topics were discussed, including:

- Formal theory [63].

- Astro-particle topics such as the CMB, dark energy, and inflation [53].

- Future facilities [33, 64, 65].

- Detector and accelerator development [66].

- Computing [67].

- Technology applications and industrial ties [68].

- Education and outreach [69].

- Diversity [70]. 


\section{Thoughts for the future}

I often hear my colleagues express frustration about the recent lack of exciting new discoveries. However, it is sometimes useful to step back and take a longer view. For example, Newtonian gravity was spectacularly successful. It survived intact for some 230 years before being supplanted by general relativity (or at least 175 years until the deviation in Mercury's perihelion was established). In contrast, the Standard Model is equally successful, but is scarcely 50 years old (and the Higgs discovery was only six years ago). If the SM had fallen quickly and easily it would not be such a great accomplishment. We should take pride in the SM and also the Standard Cosmological Model, which was developed in parallel. They will figure prominently in the history of science.

Of course, as human beings with relatively short lifetimes we have both psychological (i.e., our enthusiasm for what we are doing) and practical (e.g., funding, jobs, interest in the field by the public and especially by young potential scientists) reasons to hope for new developments. Fortunately, despite the SM successes there are strong reasons (Section 2.4) to believe that there is much still to be learned. Perhaps there will indeed be new layer(s) of physics at the (multi-)TeV scale. Or perhaps we may eventually be able to construct a New Standard Model of Nature that incorporates cosmology and extends all the way to the Planck scale. I am optimistic that one or both of these will occur. The time scale is less certain. Next week? In 20 years? In 100 years?

\subsection{Ideas (conventional and not) for new physics}

There are many ideas for possible BSM physics, including:

- Symmetries, such as supersymmetry or family symmetries.

- Compositeness or other strong dynamics, e.g., composite Higgs, dynamical symmetry breaking, or composite fermions.

- Extra dimensions, which could be "large" (compared to the Planck length of $\sim 10^{-33} \mathrm{~cm}$ ) and/or warped.

- Dark or (quasi-)hidden sectors. These could be associated with dark matter, supersymmetrybreaking, or be "random".

- Unification, such as grand unification, superstring theories, AdS/CFT, or holography.

- New dynamical ideas, such a relaxion models, nnaturalness, clockwork models, or string instantons.

- Random or environmental selection, such as in the multiverse or variations.

- Remnants, i.e., new physics that does not solve specific SM problems, but emerges more or less accidentally from an underlying theory. Typical superstring theory remnants include $Z$ 's, vector fermions, extended Higgs sectors, dark sectors, moduli, and axions [71].

- Here be dragons. Novel concepts, such as emergent interactions, dimensions, or spacetime (e.g., from quantum entanglement [63]); hidden variables; .... 


\subsection{The paradigms}

It is usually assumed that Nature (except possibly for gravity) is described by a unique quantum field theory. However, there are an infinite number of such theories, differing by the types of interactions, fields, symmetries, couplings, and masses. How does Nature choose between them? Principles such as elegance, naturalness, minimality, or "uniqueness" (within some ad hoc framework) are often explicitly or tacitly invoked, but none of these are unambiguous or compelling.

Another issue is that Nature seems to be "just right" [72]. For example, the dark energy is small enough in magnitude for structures like galaxies to form before matter becomes too diffuse or space recollapses. Similarly, the interactions and parameters of the SM seem to be fortuitously arranged to allow for the existence of multiple types of stable atoms, and for stars, etc. Yet another example is that stable planetary orbits are only possible in three large space dimensions.

Moreover, these two issues, i.e., the selection principle on the infinite landscape of field theories, and the fact that the one chosen is so suitable for our existence, must be satisfied simultaneously.

These types of questions take us somewhat out of the realm of the traditional scientific method into that of philosophy. In my opinion, however, developments in superstring theory and cosmology, and the absence (so far) of evidence for new physics relevant to the Higgs hierarchy problem and other SM shortcomings, suggest that the time is ripe to examine some of the usual paradigms (e.g. [4, 71]).

\section{Minimality or remnants}

Minimality, closely related to Occam's Razor, refers to the ansatz that any new physics should be as simple as possible and that it should solve one, or preferably more than one, SM problem.

Minimality is often a good guide, but not always so. The human brain, DNA, and the Standard Model do not appear to be minimal, and new physics might not be either. In particular, short distance theories such as superstrings, grand unification, or strong coupling compositeness can easily lead to features such as vector fermions or $Z^{\prime}$ s that "slip through the cracks" and remain light. These remnants might not solve any SM problems and are usually not minimal.

\section{Naturalness or tuning}

Naturalness has frequently been a good guide as to the existence and mass scales of new physics that could resolve fine-tuning problems. Notable examples [73] include the electron mass, $m_{\pi^{+}}-m_{\pi^{0}}$, and $m_{K_{L}}-m_{K_{S}}$, which led (or could have led) to predictions of the positron, the $\rho$ (or constituent quark), and the $c$ quark and their mass scales. Similarly, the Higgs hierarchy problem apparently requires an unnatural fine-tuning by some 34 orders of magnitude in the SM, but can be cured if there is new $\mathrm{TeV}$-scale physics such as supersymmetry, a composite Higgs, or large and/or warped extra dimensions. So far, however, ATLAS and CMS have not observed any sign of such effects. Even more telling is that the observed dark energy suggests a cosmological constant tuned by some 120 orders of magnitude compared to the Planck scale. Perhaps naturalness has failed.

A plausible alternative to naturalness is the environmental (or anthropic) explanation of finetuning, i.e., that it is required for Nature to be just right. This would make no sense if there were a unique physics selected by some non-environmental mechanism. However, it is perfectly 
reasonable if it is a consequence of a very large landscape of vacua (such as are believed to exist in string theory) and some mechanism, such as eternal inflation, to sample them [74]. Structures such as galaxies, stars, and people would then emerge only in the suitable vacua. In fact, environmental selection is probably the most plausible explanation of the small dark energy [75]. These issues will be further discussed in the next section.

Other clever dynamical mechanisms, such as clockwork theories or relaxions, have been suggested as technically natural solutions to the fine-tuning problems.

\section{Uniqueness or environment}

The uniqueness paradigm assumes that there is a unique and perhaps simple underlying theory of Nature. As commented above there are actually an infinite number of possible theories and no clear mechanism to understand which one Nature chooses. Gauge theories offer a partial solution, in that the form of the interactions is constrained up to the group, representations, coupling constant, and spontaneous symmetry breaking. However, Yukawa and scalar interactions are unconstrained unless new symmetries or principles are invoked.

There is a famous example of the failure of the uniqueness paradigm: the Mysterium Cosmographicum, in which Johannes Kepler attempted to describe the relative radii of the six known planetary orbits by nesting the Platonic solids. Kepler's theory was reasonably successful given the accuracy of the observations at the time, but we now know that this was fortuitous. There are numerous planetary systems, and their orbits are determined by initial conditions and not uniqueness. Moreover, the lucky coincidence that the Earth has favorable conditions for life is not really a coincidence - life can only develop where conditions are suitable.

The environmental paradigm is analogous to the modern understanding of the planetary orbit problem. It suggests that there may be no simple and unique explanation for some (or all?) of the features and parameters of Nature, but that rather they depend on the details of the particular vacuum. This concept is not just idle speculation: it is suggested by superstring theory, which appears to have an enormous landscape of vacua [ $\mathscr{O}\left(10^{272,000}\right)$ by one recent estimate [76]], with no known selection principle. Different vacua involve different gauge groups, hierarchies, parameters, remnants, and number of large dimensions, and only a subset would be habitable (just right). Furthermore, eternal inflation [74] could have sampled these vacua, creating a multiverse of spatially separated universes with different physics. ${ }^{9}$ Such a string multiverse could provide a reasonable anthropic explanation of some apparent fine-tunings, while other arbitrary features of Nature (e.g., the masses of the third family fermions or the neutrino mixings) could be essentially random. ${ }^{10}$

\section{Is string theory and the multiverse verifiable?}

The major problems with this string theory/eternal inflation/multiverse picture are whether it is testable? Falsifiable? Believable? Desirable? True? Scientific? Many scientists reject it on these grounds. However:

- Superstring theory is a consistent and finite theory of quantum gravity (at least at the perturbative level). The importance of this should not be forgotten.

\footnotetext{
${ }^{9}$ Variations on this idea could involve different vacua being separated in time, as different branches of a quantum wave function, etc.

${ }^{10}$ It would not necessarily mean that all aspects of the SM are arbitrary.
} 
- The multiverse concept is not ad hoc: it may eventually be shown to follow as a necessary consequence of string theory and eternal inflation.

- Some types of new physics that are allowed in field theory occur rarely or never in superstring vacua. Examples include large representations, global symmetries, and tri-fundamental representations. Their observation would go far towards falsification. Recently, similar themes have been expressed in the "swampland" conjectures (e.g., [77]) that most otherwiseconsistent effective field theories are probably inconsistent with theories of quantum gravity such as the string landscape. These conjectures are well-motivated by string constuctions.

- String remnants are common in the landscape [71], and their observation would provide positive support.

- Technical progress is possible. For example, it is currently debated whether the landscape includes many or any vacua that are metastable or de Sitter (positive energy), or how many correspond to quintessence, but these questions should essentially be settled. Similarly, theory and observations should clarify the status of eternal inflation.

- Completely new ideas may emerge. Who would have anticipated the role of the CMB for cosmology, or of DNA for paleoanthropology?

My view is that the multiverse should be taken very seriously as a possibility. Its establishment would be the sixth Copernican revolution! ${ }^{11}$

\subsection{How will we make progress?}

There are many ideas for BSM physics, and of course there could be things that no one has thought of. Fortunately, progress is anticipated on a number of fronts.

\section{The energy frontier}

The LHC program to date has been spectacular. The accelerator, detectors, computing, and theoretical support (QCD calculations, simulations, lattice calculations, ...) have all been impressive, and the program has functioned as a model of international cooperation. Physics highlights include verifications of the SM, the Higgs discovery, and numerous constraints on BSM.

The next 20 years or so will witness a large increase in luminosity [Figure 20], allowing more precise measurements of the Higgs couplings, searches for BSM, dark matter, and remnants.

On a still longer time scale there are serious proposals for much higher energy [ $\mathscr{O}(100 \mathrm{TeV})]$ $p p$ colliders (the FCC- $h h$ at CERN and the SppC in China), which would be able to probe the Higgs, naturalness, dark matter, remnants, and electroweak symmetry breaking and baryogenesis. Other possibilities at the energy frontier include the proposed HE-LHC (at $\sim 27 \mathrm{TeV}$ ), electronhadron colliders (LHeC, FCC-eh), and a muon collider (FCC- $\mu \mu$ ). However, there is no "no-lose" theorem for these proposals analogous to that at the LHC for electroweak symmetry breaking.

There is also expected to be a vigorous continuation of the heavy ion program, with implications for the QCD phases, including the quark-gluon plasma.

\footnotetext{
${ }^{11}$ The Earth is not the center of the Solar System, or of the galaxy. There are other stellar/planetary systems, and other galaxies. Ordinary matter constitutes only a fraction of the total energy density.
} 
Finally, new technologies are being pursued for detectors and accelerators, including superconducting magnet technologies, plasma wave acceleration, etc. New techniques in machine learning for data analysis are being pursued. These could also find application in studying the string landscape.

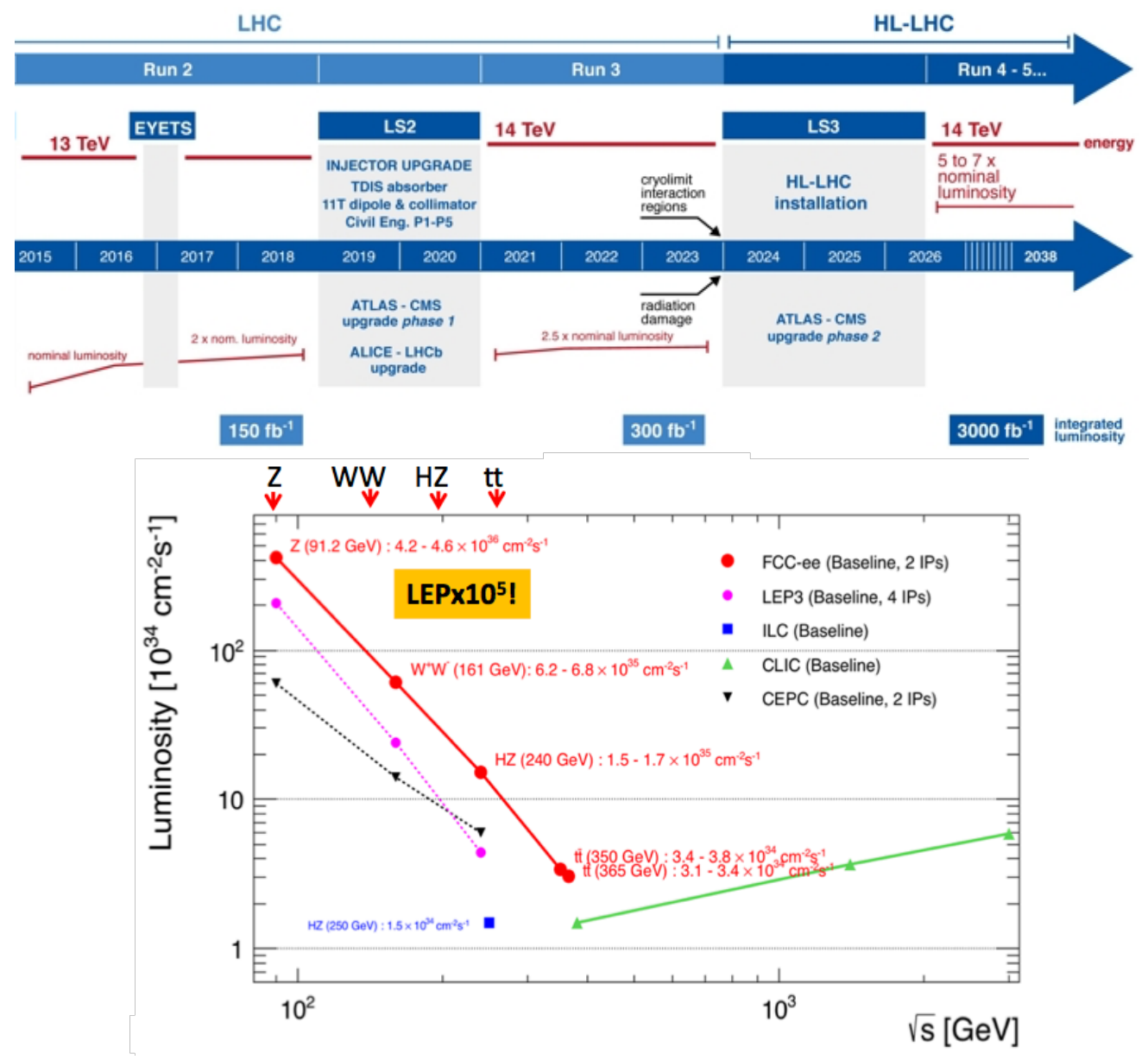

Figure 20: Top: LHC luminosity [32]. Bottom: proposed $e^{+} e^{-}$colliders [78].

\section{The precision frontier}

There are a number of proposed $e^{+} e^{-}$colliders [Figure 20]. The International Linear Collider (ILC) in Japan would initially run at $250 \mathrm{GeV}$, but could be upgraded to higher energies. The FCC-ee and CEPC are circular collider proposals that would have higher luminosity but would be limited in energy because of synchrotron radiation. These would be the first stages of the 100 $\mathrm{TeV} p p$ collider projects in CERN and China, respectively. The CLIC proposal at CERN would be a very high energy linear collider with a novel drive-beam acceleration technology. The $e^{+} e^{-}$ colliders could carry out precise studies of the Higgs and search for BSM. They could also repeat the $Z$-pole program with much higher precision, and determine the $W$ and $t$ masses more accurately.

There are many large and small flavor physics experiments, including the BELLE II and LHCb programs and smaller dedicated experiments on $g_{\mu}-2$, FCNC, kaon physics, EDMs, etc.; new 
large-scale proton decay experiments HyperKamiokande and DUNE [65]; and searches for weakly coupled, light, dark, or long-lived particles at colliders, beam dumps, AMO, and dedicated experiments.

\section{Neutrino physics}

There are numerous outstanding questions in neutrino physics. These include the details of the spectrum (leptonic $C P$ violation, mixings, the mass hierarchy) and whether it is due to a symmetry or anarchy; whether the masses are Majorana or Dirac, and their origin; light sterile neutrinos and their mixing with ordinary neutrinos; $\mathrm{keV}$-scale sterile neutrinos; electromagnetic properties and nonstandard interaction; decays; Solar physics; supernova physics; and high energy sources.

There is a large world-wide program to address these issues, including long and short baseline accelerator experiments; reactor, source, and spallation experiments; under ice and under water experiments; Solar and atmospheric neutrinos; $\operatorname{cosmology} ; \beta$ decay and related experiments; $\beta \beta_{0 v}$; and (possibly) detection of relic neutrinos.

\section{Cosmology and astrophysics}

Topics most directly connected to particle physics include inflation or alternatives; dark matter and energy; constraints on the mass, number, and interactions of ordinary and sterile neutrinos, axions and ALPs, and other exotic particles; primordial black holes, moduli, and defects (e.g., cosmic strings, domain walls, monopoles); phase transitions; and exotic matter at high temperature or density.

We are fortunate to be living in a golden age of observations in astrophysics and cosmology. Relevant programs ${ }^{12}$ include dark matter searches (direct, indirect, collider; axion and ALP searches; and dark photon searches); studies of the CMB polarization, baryon acoustic oscillations, $21 \mathrm{~cm}$ radiation, the Lyman-Alpha forest, and the DES and LSST telescopes; gravity wave interferometers and multi-messenger astronomy; high energy cosmic rays, $\bar{p}, e^{ \pm}, \gamma, v$; and very large telescopes.

\section{Realities}

There have been outstanding recent achievements in particle physics, astrophysics, and cosmology, and the technical prospects for the future are brilliant. However, we are challenged by daunting political, economic, and human resource realities. It is imperative that as a field we concentrate not only on our science, but also on outreach and education (to the public, politicians, industry, and especially young people); diversity; and spinoff technology. We must also emphasize that high energy physics (and big science generally) is a model for international cooperation.

\subsection{The quest}

It is my hope that we will eventually develop and establish a New Standard Model of Nature, incorporating physics all the way to the Planck scale, and describing the Universe on the largest scale and earliest time (or to go as far as possible towards these goals).

\footnotetext{
${ }^{12}$ Analyses are complicated by the discrepancy between local and CMB determinations of the Hubble constant.
} 
Such a lofty aspiration will require significant progress in experiment, observation, and bottomup and top-down theory, as well as ingenuity, hard work, and luck. It will also need the cooperation of Nature.

This goal is extremely ambitious. We may never achieve it, or it may take a very long time, but we should try.

\section{Thanks}

It is a pleasure to thank everybody involved for a wonderful conference. This includes the cochairs Jihn E. Kim, Un-ki Yang, and Yeongduk Kim; the program committee Young Joon Kwon, Sung Youl Choi, and Suyong Choi; as well as the International Advisory Committee, the local staff members, all of the sponsors, and especially the participants.

\section{References}

[1] S. Weinberg, A Model of Leptons, Phys. Rev. Lett. 19, 1264 (1967).

[2] A. Pais, Inward Bound: Of Matter and Forces in the Physical World, Clarendon Press, Oxford, 1986.

[3] S. Weinberg, The Quantum Theory of Fields, vol. 1, Cambridge University Press, Cambridge, 1955.

[4] P. Langacker, Can the Laws of Physics be Unified?, Princeton University Press, Princeton, 2017.

[5] P. A. M. Dirac, Quantum theory of emission and absorption of radiation, Proc. Roy. Soc. Lond. A 114, 243 (1927).

[6] J. Schwinger, ed., Quantum Electrodynamics, Dover, New York, 1958.

[7] C. N. Yang and R. L. Mills, Conservation of Isotopic Spin and Isotopic Gauge Invariance, Phys. Rev. 96, 191 (1954).

[8] R. F. Streater and A. S. Wightman, PCT, Spin and Statistics, and All That, Princeton University Press, Princeton, 2000.

[9] E. Fermi, An attempt of a theory of beta radiation. 1., Z. Phys. 88, 161 (1934).

[10] E. Noether, Invariant Variation Problems, Gott. Nachr. 1918, 235 (1918) [Transp. Theory Statist. Phys. 1, 186 (1971)] [physics/0503066].

[11] M. Gell-Mann, Symmetries of baryons and mesons, Phys. Rev. 125, 1067 (1962).

[12] Y. Nambu and G. Jona-Lasinio, Dynamical Model of Elementary Particles Based on an Analogy with Superconductivity. 1., Phys. Rev. 122, 345 (1961).

[13] J. Goldstone, Field Theories with Superconductor Solutions, Nuovo Cim. 19, 154 (1961).

[14] M. Gell-Mann, A Schematic Model of Baryons and Mesons, Phys. Lett. 8, 214 (1964).

[15] O. W. Greenberg, Spin and Unitary Spin Independence in a Paraquark Model of Baryons and Mesons, Phys. Rev. Lett. 13, 598 (1964).

[16] J. D. Bjorken, Asymptotic Sum Rules at Infinite Momentum, Phys. Rev. 179, 1547 (1969).

[17] M. Breidenbach et al., Observed Behavior of Highly Inelastic electron-Proton Scattering, Phys. Rev. Lett. 23, 935 (1969). 
[18] P. W. Higgs, Broken symmetries, massless particles and gauge fields, Phys. Lett. 12, 132 (1964).

[19] F. Englert and R. Brout, Broken Symmetry and the Mass of Gauge Vector Mesons, Phys. Rev. Lett. 13, 321 (1964).

[20] A. Salam, Weak and Electromagnetic Interactions, in Elementary Particle Theory, ed. N. Svartholm (Almquist and Wiksells, Stockholm 1968), 367-377.

[21] S. L. Glashow, Partial Symmetries of Weak Interactions, Nucl. Phys. 22, 579 (1961).

[22] G. 't Hooft, Renormalizable Lagrangians for Massive Yang-Mills Fields, Nucl. Phys. B 35, 167 (1971).

[23] S. L. Glashow, J. Iliopoulos and L. Maiani, Weak Interactions with Lepton-Hadron Symmetry, Phys. Rev. D 2, 1285 (1970).

[24] M. Kobayashi and T. Maskawa, CP Violation in the Renormalizable Theory of Weak Interaction, Prog. Theor. Phys. 49, 652 (1973).

[25] S. Schael et al. [ALEPH and DELPHI and L3 and OPAL and LEP Electroweak Collaborations], Electroweak Measurements in Electron-Positron Collisions at W-Boson-Pair Energies at LEP, Phys. Rept. 532, 119 (2013) [arXiv:1302.3415 [hep-ex]].

[26] J. Charles et al., Current status of the Standard Model CKM fit and constraints on $\Delta F=2 \mathrm{New}$ Physics, Phys. Rev. D 91, 073007 (2015) [arXiv:1501.05013 [hep-ph]].

[27] T. Carli, these proceedings.

[28] Y. Fukuda et al. [Super-Kamiokande Collaboration], Evidence for oscillation of atmospheric neutrinos, Phys. Rev. Lett. 81, 1562 (1998) [hep-ex/9807003].

[29] H. Fritzsch, M. Gell-Mann and H. Leutwyler, Advantages of the Color Octet Gluon Picture, Phys. Lett. 47B, 365 (1973).

[30] D. J. Gross and F. Wilczek, Ultraviolet Behavior of Nonabelian Gauge Theories, Phys. Rev. Lett. 30, 1343 (1973).

[31] H. D. Politzer, Reliable Perturbative Results for Strong Interactions?, Phys. Rev. Lett. 30, 1346 (1973).

[32] S. Rahatlou, these proceedings.

[33] K. Akai, these proceedings.

[34] P. Piacquadio, these proceedings.

[35] R. Averbeck, these proceedings.

[36] T. Hirano, these proceedings.

[37] L. Skinnari, these proceedings.

[38] M. Dolan, these proceedings.

[39] D. del Rey, these proceedings.

[40] S. Strandberg, these proceedings.

[41] P. Urquijo, these proceedings.

[42] E. Waheed, these proceedings. 
[43] N. Tuning, these proceedings.

[44] S. Fajfer, these proceedings.

[45] S. Mihara, these proceedings.

[46] P. Shanahan, these proceedings.

[47] S. Fang, these proceedings.

[48] E. Elouadrhiri, these proceedings.

[49] M. Yokoyama, these proceedings.

[50] S. Petcov, these proceedings.

[51] D. Garcia-Gamez, these proceedings.

[52] R. Saakyan, these proceedings.

[53] M. Hazumi, these proceedings.

[54] M. Weber, these proceedings.

[55] M. Drees, these proceedings.

[56] H. S. Lee, these proceedings.

[57] A. Kounine and S. Ting, these proceedings.

[58] Y. Semertzidis, these proceedings.

[59] S. R. Klein [IceCube Collaboration], High-energy neutrino interaction physics with IceCube, arXiv: 1809.04150 [hep-ex].

[60] H. Dujmović, these proceedings.

[61] B. P. Abbott et al. [LIGO Scientific and Virgo Collaborations], Tests of general relativity with GW150914, Phys. Rev. Lett. 116, 221101 (2016) [arXiv:1602.03841 [gr-qc]].

[62] P. Shawhan, these proceedings.

[63] T. Takayanagi, these proceedings.

[64] X. Lou, these proceedings.

[65] J. Yu, these proceedings.

[66] D. Bortoletto, these proceedings.

[67] E. Sexton-Kennedy, these proceedings.

[68] P. Riedler, these proceedings.

[69] K. Shaw, these proceedings.

[70] S. Yacoob, these proceedings.

[71] J. Halverson and P. Langacker, TASI Lectures on Remnants from the String Landscape, PoS TASI 2017, 019 (2018) [arXiv:1801.03503 [hep-th]].

[72] A. N. Schellekens, Life at the Interface of Particle Physics and String Theory, Rev. Mod. Phys. 85, 1491 (2013) [arXiv:1306.5083 [hep-ph]]. 
[73] G. F. Giudice, Naturally Speaking: The Naturalness Criterion and Physics at the LHC, [arXiv:0801.2562 [hep-ph]].

[74] A. Linde, A brief history of the multiverse, Rept. Prog. Phys. 80, 022001 (2017) [arXiv:1512.01203 [hep-th]].

[75] S. Weinberg, The Cosmological Constant Problem, Rev. Mod. Phys. 61, 1 (1989).

[76] W. Taylor and Y. N. Wang, The F-theory geometry with most flux vacua, JHEP 1512, 164 (2015) [arXiv:1511.03209 [hep-th]].

[77] T. D. Brennan, F. Carta and C. Vafa, The String Landscape, the Swampland, and the Missing Corner, PoS TASI 2017, 015 (2017) [arXiv:1711.00864 [hep-th]].

[78] A. Blondel, these proceedings. 